\title{
Applying W3C PROV to Express Geospatial Provenance at Feature and Attribute Level
}

\author{
Joan Masó $^{1(\bowtie)}$, Guillem Closa ${ }^{1}$, and Yolanda Gil ${ }^{2}$ \\ ${ }^{1}$ Center for Ecological Research and Forestry Applications, \\ 08193 Cerdanyola del Vallès, Spain \\ joan.maso@uab.cat,g.closa@creaf.uab.cat \\ 2 Information Sciences Institute, University of Southern California, \\ 4676 Admiralty Way, Marina del Rey, CA 90292, USA \\ gil@isi.edu
}

\begin{abstract}
This paper presents the application of PROV to geospatial data. In particular, it is applied to the vector model, where geospatial phenomena are represented as a collection of individual objects (called features) that are described with a lot of geographical (point, lines, polygons, etc.) and nongeographical (names, measures, etc.) properties (sometimes called attributes). We present an approach to describe in W3C PROV the distributed data sources and the processes involved in the generation/revision of a geospatial dataset.
\end{abstract}

Keywords: $\mathrm{W} 3 \mathrm{C} \cdot$ Geospatial provenance $\cdot \mathrm{RDF} \cdot$ Distributed environments

\section{Geospatial Provenance in W3C PROV}

This paper presents an application of PROV to describe geospatial provenance generated on a distributed environment and encoded in RDF. To apply PROV in the geospatial domain it is important to identify the different elements that PROV provides and map them to geospatial concepts. The PROV Data Model relies on the definition of entities, activities, and agents and the relations among them [1]. In the geospatial world information is stored in features. A feature is described as a set of geographical and non-geographical properties (sometimes called attributes). Features can be tangible, such as rivers; or an abstract concept, such as political boundaries [2]. Geographical properties are the position and shape of a feature that can be expressed as a point a line or a polygon (as a sequence of coordinates). Non-geographical property can be for example the name of a river.

Each geospatial feature can be considered a PROV entity, and we refer to the provenance associated with it as feature-level provenance. Geospatial features are grouped in collections of features. A feature collection (called datasets) can also be considered an entity in PROV (in fact, an entity collection), the dataset-level provenance. In many occasions, feature properties are processed with different techniques and can come from different origins. In that case, their attributes (also considered entities), have their own provenance, the attribute-level. Therefore, we capture 
geospatial provenance information at three different levels of granularity. In a Geospatial Information System (GIS) context, activities are executions that create and manipulate datasets, features and attributes (such as buffer generation or conflation execution). Activities can be executed in a GIS platform or a web processing service. Each of the individual activities can be associated with a general process that can be represented as a PROV Plan. The GIS platform or the web processing service can be considered a plan collection. Another important aspect to consider is identifying the PROV agents, that can be either responsible of an individual process execution (activity) or be the developer of a general process (plan).

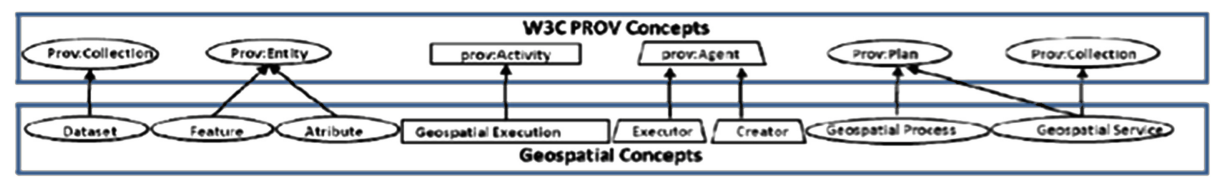

Fig. 1. The correspondence between $\mathrm{W} 3 \mathrm{C}$ PROV and Geospatial concepts

We use the RDF subclass mechanism to map geospatial elements to PROV classes, as shown in Fig. 1. In addition, we use the Turtle notation here to show examples, with geospatial concepts shown with the "ows" namespace. For example:

ows:Feature rdfs:subclassof prov:Entity .

\section{W3C PROV Relations Applied to Geospatial Provenance}

In our application, we can derive a new dataset by combining two data sources through an activity requested by an agent. A new features wasGeneratedBy the combination process and wasDerivedFrom the two initial features. In RDF:

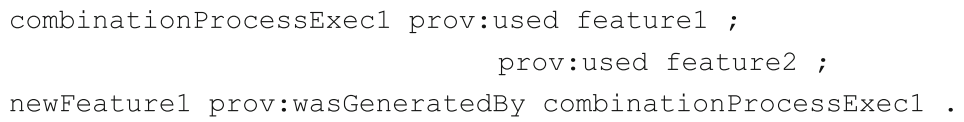

At the same time, the geospatial process wasAssociatedWith an agent, which can be considered the author (wasAttributedTo) of the new features:

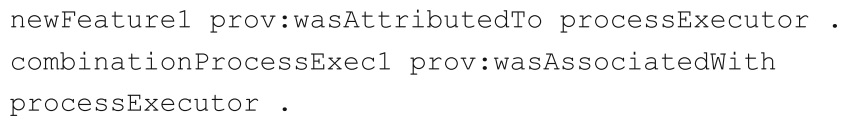

We can associate the individual execution with the generic GIS tools used (plan) and with the developer of the tools:

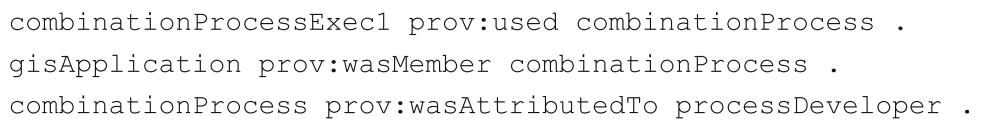


These examples describe feature-level provenance, but the same relations can be used at dataset or attribute level. This is an excerpt of the provenance for 2 attributes of newFeature1:

newPosition1 prov:wasDerivedFrom position1 .

\section{W3C PROV and RDF to Express Geospatial Provenance}

The Open Geospatial Consortium has defined a standard for geospatial processing on the web called Web Processing Service (WPS) [3]. As a use case, we have defined how to implement a distributed process for the conflation of two datasets as a WPS process. In the geospatial field, a conflation process is defined as the process of combining geographic information from overlapping sources so as to retain accurate data, minimize redundancy, and reconcile data conflicts [4]. In our case, a U.S. Geological Survey (USGS) dataset was enhanced with Open Street Map (OSM) dataset by adding new features or updating the geometry or other attributes. The conflation process uses a matching and enrichment algorithm controlled by a distance parameter (that acts as a threshold for the conflation step where features in the OSM beyond that distance will not be conflated with a given USGS feature). Dataset-level provenance information captures the purpose of each parameter, the name of the authors of the data and the processes involved, the date and time of both the process development and the execution. During conflation, some completely new features are added, and for them feature-level provenance is provided. Other features are conflated by modifying only the geometrical property (location) or the non-geometrical properties, and a more detailed and granular attribute-level provenance is used.

\section{Conclusions}

PROV can be used to express, store and query geospatial information. The model presented defines geospatial entities and captures provenance at 3 levels of granularity. The RDF encoding of PROV can express well the provenance of the geospatial objects, roles and relations with a uniform approach. PROV was found more flexible, easier to understand, and more compact than ISO19115 XML encoding that is traditionally used in the geospatial community.

Acknowledgments. This work is a continuation of the GeoViQua FP7 project, and organized and funded by the Open Geospatial Consortium as part of the OWS10 Interoperability Experiment. It was also supported in part by the US Air Force Office of Scientific Research with grant FA9550-11-1-0104. 


\section{References}

1. Moreau, L., Missier, P. (eds.): PROV-DM: The PROV Data Model. World Wide Web Consortium (2013)

2. Lake, R., et al.: Geography Mark-Up Language. Wiley, Chichester (2004). ISBN 978-0470871546

3. Schut, P.: OpenGIS Web Processing Service Version 1.0.0. OGC 05-007r7 (2007)

4. Chen, C.-C., et al.: Automatically and accurately conflating raster maps with orthoimagery. Geoinformatica 12(3), 377-410 (2008). doi:10.1007/s10707-007-0033-0 